Résumés des conférences et travaux

\title{
Jean-Louis Ferrary (1948-2020)
}

\section{Ségolène Demougin}

\section{OpenEdition}

\section{Journals}

Édition électronique

URL : https://journals.openedition.org/ashp/4934

DOI : $10.4000 /$ ashp.4934

ISSN : 1969-6310

Éditeur

Publications de l'École Pratique des Hautes Études

\section{Édition imprimée}

Date de publication : 1 septembre 2021

Pagination : XXI-XXV

ISSN : 0766-0677

\section{Référence électronique}

Ségolène Demougin, "Jean-Louis Ferrary (1948-2020) », Annuaire de l'École pratique des hautes études (EPHE), Section des sciences historiques et philologiques [En ligne], 152 | 2021, mis en ligne le 14 juin 2021, consulté le 15 juin 2022. URL : http://journals.openedition.org/ashp/4934 ; DOI : https://doi.org/ 10.4000/ashp.4934 


\section{JEAN-LOUIS FERRARY}

(1948-2020)

I

EST douloureux de rendre l'hommage qu'il mérite à un ami de longue date, qui était aussi un collègue et un associé dans maints programmes de recherche. JeanLouis Ferrary nous a quittés le matin du 9 août 2020, des suites d'une longue maladie. L'ironie du fatum a voulu que l'origine du mal vienne d'une chute, il y a longtemps, dans une bibliothèque prestigieuse.

Comment caractériser la personnalité de Jean-Louis Ferrary? Qu'on me permette ici de citer Bossuet dans le Sermon sur la mort : « Entre toutes les passions de l'esprit humain, l'une des plus violentes, c'est le désir de savoir ». Celui-ci a guidé toute la vie de J.-L. Ferrary, aidé par d'éminentes qualités, tant humaines qu'intellectuelles : s'il inspirait de la crainte à certains - mais timor Domini initium sapientiae -, en réalité, il était bienveillant, généreux et ne pratiquait pas la méchanceté universitaire. Doté d'une grande curiosité intellectuelle - et c'est la première vertu du savant -, et d'une immense culture qui lui faisait dépasser largement les limites chronologiques de sa spécialité, il était renommé pour son érudition sans failles, sa rigueur, sa probité, son esprit critique qui lui faisait préférer en tout la vérité. Et ces qualités se sont retrouvées dans toutes ses entreprises.

Ses amis n'oublieront pas non plus son grand courage lors des premières atteintes de la maladie qui devait finir par l'emporter : il se soumit sans broncher aux traitements sévères et nécessaires, en espérant une rémission définitive; mais le destin ne l'a pas voulu.

\section{Les vertes années}

Né à Orléans le 5 mai 1948, Jean-Louis Ferrary appartenait à ce que les sociologues définissent comme « une honorable famille des classes moyennes ». Dans sa famille, il connut une situation qui rebute en général l'enfance; il resta enfant unique, ce qui fut, dans un sens bénéfique, comme il le disait lui-même, pour expliquer son goût pour la lecture; quand nous en parlions, il me disait « que peut faire pour s'occuper un gamin qui n'a ni frère ni sœur? ». Cette addiction à la lecture et au livre l'amena plus tard, quand il fut plus grand, à la bibliophilie et à la constitution d'une bibliothèque extraordinaire et précieuse.

Cet enfant, intelligent et solitaire, s'épanouit dans l'école républicaine, et parvenu, summa cum laude, au baccalauréat, il se dirigea, sans coup férir, vers les classes préparatoires, celles du lycée Louis-le-Grand en l'espèce, et l'entrée à l'École normale s'ensuivit naturellement en 1967.

\section{La naissance d'un maître}

Attiré depuis longtemps par l'Antiquité classique, J.-L. Ferrary avait décidé d'y chercher son accomplissement professionnel et d'en faire son champ de recherche. 
Ce choix se marqua par son rang de cacique de l'agrégation de lettres classiques en 1970. À l'époque, il penchait fortement - disons qu'il était séduit - par le monde hellénique; mais il fut arrêté dans son élan par le directeur d'alors de l'École normale qui le dissuada de se présenter au concours de l'École d'Athènes. Tant pis pour les études grecques qui perdirent ainsi un maître, et tant mieux pour les études romaines qui en trouvèrent un. Mais J.-L. Ferrary sut rattraper plus tard l'hellénisme. Finalement, ce n'est pas l'Acropole qui l'accueillit, mais le palais Farnèse. C'est dans ce cadre prestigieux qu'il parfit sa formation et qu'il entreprit les travaux préliminaires à sa thèse d'état. Et c'est là aussi qu'il fit ses premières et dernières expériences d'archéologie sur le terrain.

De retour à Paris, Jean-Louis Ferrary commença sa carrière proprement universitaire à l'institut de latin de l'université Paris IV de la fin de 1976 à 1989; l'année 1987 marqua l'achèvement et la soutenance sa thèse d'État, thèse publiée en 1988 sous le titre: Philhellénisme et impérialisme : aspects idéologiques de la conquête romaine du monde hellénistique, de la seconde guerre de Macédoine à la guerre contre Mithridate, et rééditée en 2014. Dans ce livre, où enfin le Romain retrouvait le Grec, s'épanouit une vision nouvelle des relations intellectuelles et politiques entre Rome et le monde hellénistique; c'est à dessein que j'adopte cet ordre. On me permettra de citer ici Aldo Schiavone : « ce grand livre... capable de faire apparaître tout un ensemble de connexion entre droit, politique et philosophie, sur lequel on avait jusqu'alors glissé ».

\section{La maturité rayonnante}

Cette somme des premières grandes recherches de Jean-Louis Ferrary portait déjà en elle la diversité des nouvelles orientations, l'approfondissement de celles-ci et les intérêts pour de nouveaux champs intellectuels. Il faut bien dire que ces nouvelles et puissantes activités de J.-L. Ferrary furent grandement aidées par son entrée dans ce qui était encore la $\mathrm{IV}^{\mathrm{e}}$ section, dont il était élève diplômé depuis 1973 : recruté d'abord comme chargé de conférence par Claude Nicolet entre 1983 et 1989, il devait être élu directeur d'études en 1989, avec une direction d'études intitulée définitivement « Histoire des institutions et des idées politiques dans le monde romain ». Ce titre signalait aussi l'élargissement de certains des intérêts de J.-L. Ferrary. Cependant, dans la droite ligne de ses premières recherches, il ne quitta jamais les études sur Rome et le monde grec, et les rapports compliqués ente les cités hellénophones et la puissance latine.

Par ailleurs, Jean-Louis Ferrary accorda toujours la plus grande attention au droit public romain et aux institutions romaines. En témoigne ainsi sa participation au grand programme international lancé et dirigé par Michael Crawford, dont les résultats furent publiés sous le titre de Roman Statutes à Londres en 1996. Cette entreprise conduisit J.-L. Ferrary à concevoir un nouveau programme de recherches consacré à la réédition et surtout à la rénovation du livre de Giovanni Rotondi, Leges publicae populi Romani, paru en 1912. Réunissant là encore une équipe internationale, dont les réunions annuelles permettaient d'élaborer en réalité un tout nouveau volume, 
J.-L. Ferrary sut prendre aussi le tournant de la modernité en décidant de créer une banque de données, baptisée LEPOR, et à laquelle il continuait de travailler, en dépit des aléas de sa santé. Enfin, dernière entreprise, il s'associa avec Dario Mantovani, professeur de droit romain alors à l'université de Pavie, pour organiser, dans le cadre du Cedant (Centre d'études et de recherches sur les droits antiques), le collège de droit romain, avec une session annuelle en janvier, consacré à un thème juridique et à la formation de jeunes chercheurs. Jean-Louis Ferrary participa ainsi à de nombreuses sessions de ce programme, et aux publications qui l'accompagnaient.

Enfin il patronna la création d'une école doctorale sur les institutions et le droit romain, organisée par l'École française de Rome, et dont la mort le priva d'assister à la première session qui eut lieu en septembre 2020.

Sur ce chemin, Jean-Louis Ferrary rencontra une autre discipline, l'épigraphie, qu'il faut comprendre ici surtout comme l'épigraphie juridique, section bien particulière de ce domaine, et qui permettait encore à J.-L. Ferrary de retrouver le domaine grec. En dehors de sa participation aux Lois romaines éditées par M. Crawford, il faut joindre, en dehors de ses nombreux articles, et, surtout, ses contributions non seulement à LEPOR, mais aussi à l'édition de cette lex sur le portorium de la province d'Asie, cette loi douanière qui transforma les conceptions jusque-là admises de l'organisation financière de cette partie du monde romain. Dans le domaine de l'épigraphie grecque, on n'oubliera pas, surtout, le legs qu'il reçut de $\mathrm{M}^{\mathrm{me}}$ Robert, la charge de publier Les mémoriaux de délégations du sanctuaire oraculaire de Claros, d'après la documentation conservée dans le Fonds Louis Robert (Académie des inscriptions et belles-lettres), édition qu'il acheva en 2014.

Mais les intérêts de J.-L. Ferrary étaient multiples; il s'illustra aussi dans d'autres domaines, où sa suprématie ne faisait pas de doute, tout d'abord l'histoire politique de la Rome républicaine et augustéenne, puis l'histoire des idées politiques, auxquelles il consacra de nombreux travaux où, prenant en quelque sorte la succession de Claude Nicolet, il ne négligea pas l'œuvre de Cicéron.

Enfin, et c'était un peu son jardin personnel, il s'intéressa à l'historiographie de sa discipline, en se penchant non seulement sur les humanistes antiquaires, mais aussi sur nombre de grands érudits des derniers siècles.

La curiosité intellectuelle de Jean-Louis Ferrary, la hauteur de sa pensée et la rigueur d'une érudition sans failles expliquent l'abondance de sa bibliographie, qui compte plus de 150 articles, quatre ouvrages majeurs, trois recueils d'articles qui doivent être complétés par deux autres volumes sous presse en Italie et en Grande Bretagne, et de nombreuses directions et co-directions de volumes. D'ailleurs, il avait noué des liens très forts avec ses collègues anglais et italiens. J.-L. Ferrary était un homme de l'écrit, et même dans les derniers mois, il continuait à travailler malgré les tourments de la maladie. Une bibliographie complète de ses publications a été élaborée par Denis Rousset et Philippe Moreau, ses amis, et se trouve facilement sur le site internet qui lui a été consacré (http://souvenir-de-j-1-ferrary.fr), et qui le fait rester avec nous. Elle se trouvera aussi dans un petit livre qui doit être publié prochainement en sa mémoire par les Belles Lettres, maison d'édition à laquelle il était très attaché, et où il dirigea la prestigieuse section latine de la Collection des universités de France. 


\section{Les vertus et les honneurs}

Il ne faudrait pas voir en Jean-Louis Ferrary un érudit isolé dans sa tour d'ivoire, sans réels contacts avec le monde de la recherche. Il a toujours considéré que l'universitaire qu'il était se devait d'animer la recherche, de participer aux tâches nécessaires mais parfois rebutantes de l'administration, et d'assurer des responsabilités éditoriales.

Ainsi, il sut toujours accueillir les jeunes chercheurs, qu'ils travaillent ou non sous sa direction, et leur inculquer les "bonnes manières " nécessaires à toute carrière scientifique de qualité. Il ne refusa pas les directions d'équipe : directeur d'une imposante UMR d'histoire romaine, entre 1995 et 2005, puis à nouveau en 2009, il mena à bien la fusion de deux grandes bibliothèques parisiennes d'Antiquité, la bibliothèque Gernet, et la bibliothèque Glotz, réunies à l'Institut national d'histoire de l'art, et fut aussi l'un des artisans de la fusion des équipes de recherche correspondantes. À l'École pratique, où il ne refusa pas de payer de sa personne dans les conseils et comités de la section, il fut aussi assesseur à la recherche de 2010 à 2011 et membre du conseil scientifique de l'établissement, entre 2010 et 2014. À l'École française de Rome, il fut à la fois membre du conseil scientifique à partir de 2011, et président du conseil d'administration de 2011 à 2017. Pour en rester aux institutions françaises, il était membre du conseil scientifique de l'Institut de droit romain de Paris. On ne saurait oublier ici les présidences qu'il assuma de nombreuses associations et sociétés savantes, comme l'Association Guillaume-Budé (2011-2019) ou la Société d'études épigraphiques sur Rome (2005-2006).

Cependant, la réputation scientifique de J.-L. Ferrary le fit aussi rechercher par de nombreuses institutions universitaires et scientifiques internationales, d'abord comme membre de leur conseil scientifique, comme à Florence, Pavie, Pise, en Italie, le Thesaurus Linguae Latinae à Munich, et enfin à la fondation Hardt dont il devint le président en 2017.

Jean-Louis Ferrary était particulièrement attaché à la diffusion des sciences historiques et philologiques : en témoignent les nombreuses entreprises éditoriales, où il œuvra durant des années : co-directeur de la Revue de philologie de 1992 à 2007, il dirigea, comme on l'a déjà dit, à partir de 2001 la série latine de la Collection des universités de France, où son absence va se faire cruellement sentir. Il fut aussi extrêmement sollicité pour appartenir à des comités de lecture ou à des comités scientifiques de revues, surtout en Europe : Cahiers du centre Glotz et Revue de philologie, Cahiers de l'humanisme (en France), Mediterraneo Antiquo, Athenaeum, Rivistà di fililogia e d'instruzione classica (en Italie), Annaeus (en Espagne) ainsi que Scripta Antiqua et Vetsnik drevnej istorii (à Moscou). En plus de cette activité éditoriale, J.-L. Ferrary fut aussi un éveilleur intellectuel, participant très activement à la diffusion de la recherche : je renonce ici à citer ici les vingt-huit académies et établissements qui l'accueillirent pour des séjours ou des conférences, en insistant toutefois sur son passage à l'Institute for Advanced Study à Princeton en 1993, et, entres autres, sur sa conférence d'ouverture en 2016, pour la Society for the Promotion of Roman Studies.

Cette activité scientifique remarquable et multiforme l'amena à l'honneur d'être élu, en 2005, à l'Académie des inscriptions et belles lettres, au fauteuil de Maurice 
Euzennat. Il déploya, dans cette compagnie, la même énergie au service de l'Antiquité classique. La reconnaissance de ses pairs s'exprima par son élection à la présidence de l'Académie, en 2018, jointe à celle de l'Institut de France, la même année.

\section{L'homme n'est pas seulement un pur esprit}

Le rappel que je viens de faire des multiples activités et du rôle éminent de JeanLouis Ferrary pourraient faire croire qu'il ne s'est consacré qu'à la science et qu'il a toujours appartenu au cercle exclusif des savants austères. En réalité il n'en est rien. Un seul domaine l'a toujours rebuté, celui de l'économie domestique. Contraint, par les rigueurs du confinement de cette année 2020, à acheter un four à micro-ondes, il s'en étonnait encore et restait dubitatif devant cette petite merveille de la technique contemporaine. Mais, par ailleurs, tout l'intéressait : tout d'abord les beaux-arts et surtout la peinture, d'où ses nombreux voyages privés dans tous les musées d'Europe et d'ailleurs. Mélomane, il fréquentait l'Opéra de Paris et se réjouissait de la reprise prochaine du Ring de Wagner, alors qu'un destin cruel l'a privé de ces représentations. Enfin, grand liseur depuis son enfance, il s'intéressait de près à la littérature, celle du temps passé comme celle de son temps, et a développé une véritable addiction aux romans policiers de toutes nationalités; ce dépaysement nécessaire, lui faisait retrouver le droit et les pratiques juridiques. Et surtout, J.-L. Ferrary était doué d'un grand humour qui lui permettait d'affronter les difficultés, et même les déconvenues de la vie; il lui permettait d'affronter, avec ses amis, les traitements difficiles occasionnés par son état de santé. Nul n'oubliera ses grands éclats de rire.

On me permettra une conclusion brève, car on entendra encore beaucoup parler de Jean-Louis Ferrary. Nous avons perdu un très grand savant, un collègue attentionné et un ami délicat, toujours soucieux de l'avenir de sa discipline, un administrateur hors pair. Sa carrière illustre à merveille ce que Cicéron disait dans le De Senectute $(\mathrm{V})$ : « Ce n'est point par la force, la prestesse ou l'agilité du corps, que les grandes choses s'accomplissent, mais par le conseil, l'autorité, la sage maturité ». J.-L. Ferrary les a accomplies, ces grandes choses et toute sa vie en témoigne.

Loin des formule stéréotypées qu'on entend trop, comme « il va nous manquer, il nous manque déjà », qui sont plutôt le gage de l'oubli, je préfère dire qu'avec la rigueur de sa pensée et la pérennité de son œuvre, Jean-Louis Ferrary nous accompagne toujours. 